provide equitable services that are people-centered, accessible, acceptable and affordable.

It is essential to address the barriers, improve the quality of care and provide continuous training for health professionals. Moreover, women living with HIV need to be made aware of their rights and empowered to benefit themselves of these offered services and health professionals should help them understand their reproductive goals, while safeguarding their human rights.

Disclosure No significant relationships.

\section{S18.4 ENGAGING CULTURALLY AND LINGUISTICALLY DIVERSE POPULATIONS IN HIVISTI SERVICES: CHALLENGES AND OPPORTUNITIES}

Shailendra Sawleshwarkar*. University of Sydney, Australia, Faculty of Medicine and Health, Parramatta, Australia

\subsection{6/sextrans-2019-sti.84}

Cultural and linguistic diversity in many high-income countries including Australia is increasing, with more than one in four Australians being born overseas. Culturally and linguistically diverse populations (CALD) or ethnic minorities are disproportionately affected by HIV and sexually transmitted infections (STIs) in many countries with universal access to healthcare. Studies from the Australia, UK, USA and Canada suggest disparities in sexual health outcomes in CALD populations which persist even when controlling for sexual behaviour. Understanding service delivery issues for CALD populations is critical to the development and implementation of effective public health policy and health services. Enhanced HIV testing, HIV pre-exposure prophylaxis and treatment as prevention have been very effective in interrupting HIV transmission but people from CALD backgrounds especially men who have sex with men (MSM) continue to face difficulties in accessing these interventions. Lower knowledge of HIV risk factors, low perception of risk, stigma, and risky sexual risk behaviors may increase risk of HIV and STIs among CALD populations. Lack of awareness among healthcare workers regarding issues affecting CALD population also contributes to the challenges in providing effective health services. In the context of changes in HIV prevention landscape from condom promotion to biomedical approaches, sexual health services will benefit from taking culturally and linguistically sensitive approach in engaging and delivering HIV and STI care. Tailored approaches that effectively address cultural, language and gender issues to address inequalities in access and improve linkage, engagement and retention in care are needed for several CALD sub-populations, including MSM, sex workers, people who inject drugs, international students, refugees, and women. Failing to address these issues may lead to ongoing high rates of HIV transmission in these CALD subpopulations and have significant impact on achieving the goal of ending the AIDS epidemic by 2030.

Disclosure No significant relationships.
S19 - GLOBAL PUBLIC HEALTH APPROACHES TO GONORRHEA PREVENTION AND THE THREAT OF ANTIBIOTIC RESISTANCE

\author{
Wednesday, July 17, 2019 \\ 1:45 PM - 3:15 PM
}

\section{S19.1 PERSPECTIVES FROM NORTH AMERICA}

Karen Schlanger*. Centers for Disease Control and Prevention, Atlanta, USA

\subsection{6/sextrans-2019-sti.85}

Background As in other regions, rates of gonorrhea in North America continue to rise, while Neisseria gonorrhoeae $(\mathrm{Ng})$ antimicrobial susceptibility, and particularly macrolide susceptibility, has declined. These patterns have heightened concerns about emerging resistance to the only remaining recommended treatment regimen and the prospect of untreatable gonorrhea. Methods In this session, available data on $\mathrm{Ng}$ antimicrobial susceptibility trends in North America will be reviewed, with a focus on recent trends in cephalosporin, macrolide, and fluoroquinolone susceptibility. Public health responses, such as United States outbreak preparedness and response activities, will be explored.

Results Although the percentage of $\mathrm{Ng}$ isolates with reduced cephalosporin susceptibility has declined in recent years in the United States and Canada, the percentage with reduced azithromycin susceptibility has increased in both countries. Highlevel azithromycin resistant strains have been identified in the United States, and an isolate with a high ceftriaxone minimum inhibitory concentration $(1 \mu \mathrm{g} / \mathrm{ml})$ was identified in Canada (2017). Data from Mexico are limited. In addition to robust surveillance in the United States and Canada, the United States has implemented efforts to enhance rapid detection and response to $\mathrm{Ng}$ resistance.

Conclusions Robust surveillance and public health engagement are critical to address the growing threat of $\mathrm{Ng}$ resistance.

\section{S19.2 PERSPECTIVES FROM BRAZIL AND LATIN AMERICA}

Adele Benzaken*. Fundação de Medicina Tropical Doutor Heitor Vieira Dourado, Manaus, Brazil

\subsection{6/sextrans-2019-sti.86}

Global emergence of resistance to extended-spectrum cephalosporins (ESCs) cefixime and ceftriaxone is alarming scientific and healthcare communities. Following cases in Japan, a case was reported last year in England - and a Ceftriaxone-Resistant Multidrug-Resistant Neisseria gonorrhoeae (NG) was reported in Singapore in 2019. In Latin America, first isolates with reduced sensitivity to ceftriaxone were reported in 